\title{
Methodologies for Intelligent Systems
}

\author{
Guest Editor's Introduction
}

It is a great pleasure to present a selection from the best papers of the 18th International Symposium on Methodologies for Intelligent Systems (ISMIS 2009) held in Prague, Czech Republic, September 14 17. ISMIS is an established and prestigious conference for exchanging the latest research results in building intelligent systems. Held twice every three years, the conference provides a medium for exchanging scientific research and technological achievements accomplished by the international community. The scope of ISMIS is intended to represent a wide range of topics on applying Artificial Intelligence techniques. The following major areas were selected as focus areas of ISMIS 2009 conference: knowledge discovery and data mining, text mining, machine learning, applications of intelligent systems, logical and theoretical aspects of intelligent systems, information processing, agents, complex data, general AI, and uncertainty. The conference is intended to attract individuals who are actively engaged both in theoretical and practical aspects of intelligent systems.

The contributed 60 papers were selected from 111 full draft papers by the international program committee with 77 members from 23 countries and by 33 external referees. In addition, four plenary talks were given by Heikki Mannila, Gerhard Widmer, Maria Zemankova and Jozef Kelemen. The plenary talks concerned hot topics relevant to ISMIS 2009. The First International Workshop on Topological Learning organized by Michal Aupetit, Hakim Hacid and Djamel A. Zighed was co-located with ISMIS 2009. The ISMIS 2009 conference was organized by the Laboratory for Intelligent Systems, University of Economics, Prague, Czech Republic.

The authors of the selected papers were invited to submit substantially enhanced versions of their papers to this special issue, each paper was reviewed and if necessary revised by the authors. The six selected papers cover a variety of current theoretical approaches to building intelligent systems. The paper "Solving Sequential Planning Problems via Constraint Satisfaction" by Roman Barták and Daniel Toropila deals with planning as constraint satisfaction problem. The authors show how current constraint models of sequential planning problems can be enhanced in various directions. "A framework for iterated belief revision using possibilistic counterparts to Jeffrey's rule" by Salem Benferhat, Henri Prade, Didier Dubois and Mary-Anne Williams analyses the expressive power of possibilistic approach to belief revision in intelligent systems. The paper "Interval-Valued Fuzzy Galois Connections: Algebraic Requirements and Concept Lattice Construction" by Yassine Djouadi and Henri Prade deals with the situation, where the degree of fuzzy relations between objects and their properties is expressed by inter- 
vals rather than exact values. "Fuzzy Clustering for Semantic Knowledge Bases" by Floriana Esposito, Claudia d'Amato and Nicola Fanizzi focuses on the problem of clustering resources contained in knowledge bases represented by multi-relational languages that are typical for the Semantic Web. The paper "Autonomy-Oriented Search in Dynamic Community Networks: A Case Study in Decentralized Network Immunization" by Jiming Liu, Chao Gao and Ning Zhong handles network immunization as a method that can prevent virus propagation in complex dynamically-evolving networks. The authors examine the performances of an autonomy-oriented distributed search strategy for tackling such networks. "Identifying Prime Implicate Branches in Reduced Implicate Tries" by Neil V. Murray and Erik Rosenthal introduces reduced implicate tries, a tree-like data structure used for knowledge compilation and shows how to find prime implicates in this structure.

We wish to express our thanks to all authors for the excellent quality of their papers. We also thank to the reviewers of these papers for their comments.

Special issue editors

Petr Berka, Tapio Elomaa

ISMIS 2009 Program Co-Chairs

Jan Rauch

ISMIS 2009 Conference Chair

Zbigniew Ras

ISMIS 2009 General Chair 